\title{
ADHD with Co-Occurring Depression/Anxiety in Children: The Relationship with Somatic Complaints and Parental Socio-Economic Position
}

\author{
Maria Jensberg Leirbakk ${ }^{1,2 *}$, Jocelyne Clench-Aas ${ }^{2}$ and Ruth K Raanaas ${ }^{1,2,3}$ \\ ${ }^{1}$ Department of Plant Sciences, Norwegian University of Life Sciences, Norway \\ ${ }^{2}$ Department of Landscape Architecture and Spatial Planning, Norwegian University of Life Sciences, Norway \\ ${ }^{3}$ Senior Scientist, Division of Mental Health, Norwegian Institute of Public Health, Norway
}

\begin{abstract}
Attention-Deficit/Hyperactivity Disorder (ADHD) and depression/anxiety are often comorbid in children, and consequences of ADHD are more detrimental in lower socioeconomic levels. The aim of this study was to compare co-occurrence of $A D H D$ and depression/anxiety with $A D H D$ alone and depression/anxiety alone when the outcome measure is somatic complaints. Additionally, we examined whether low parental Socio- Economic Position (SEP) were related to occurrence of ADHD alone, depression/anxiety alone, and co-occurrence. The sample consisted of 12,900 parents who participated in a cross-sectional health survey that included Strengths and Difficulties Questionnaire (SDQ) and impact supplement. Socio-economic factors were dichotomized into low versus average/high parental socio-economic position (SEP), abdominal pain, neck pain and headache were categorized as somatic complaints. Parents described $58 \%$ of the children with ADHD as comorbid with depression/anxiety. Children with ADHD only report somatic complaints when thedisorder is co-occurring with depression/anxiety. The prevalence of ADHD, depression/ anxiety, or a combination of the two disorders in children, is higher when parents have a low socio-economic position. Increased awareness of the heterotypic nature of ADHD is needed, especially when interpreting somatic complaints. There is a pattern of an inverse relationship between mental health and socio-economic position in children when measured by ADHD and depression/anxiety.
\end{abstract}

Keywords: Attention deficit/hyperactivity disorder; Behavioural childhood disorder; Depression; Anxiety; Emotional disorders; Somatic complaints; Psychiatric symptom; Socio-economic status

\section{Introduction}

Attention deficit/hyperactivity disorder (ADHD) is the most common diagnosed neurodevelopmental disorder. Globally ADHD is expected to affect $3-5 \%$ of children, boys four times more frequently than girls [1]. The disorder is primarily characterized by a co-existence of problems of attention and hyperactivity, where the outcomes are difficulties controlling attention, concentration and impulsivity [1]. There is a genetic liability where the risk of a child being diagnosed with ADHD increases with $57 \%$ when a parent is diagnosed ADHD, and $32 \%$ if a sibling is diagnosed ADHD [2]. The prevalence of the disorder is higher in children from low parental socio-economic positions (SEP) [3]. In a recent review by Danckaerts et al. [4]. ADHD is associated with poor academic achievement, family- and peer- related problems and co-occurrence of other disorders. The long-term outcome of children with ADHD indicates an increased risk for criminal activity and substance abuse. The disorder is complicated by its heterogeneity, and evidence suggests high levels of comorbidity between ADHD and conduct (30-50\%), oppositional defiant disorder (40-50\%), depression and anxiety disorder (15-75\%) [5]. Both ADHD and depression/ anxiety are associated with significant impairments. The estimated prevalence of depression and anxiety under the age of 13 is $2.2-6.56 \%$ $[5,6]$. Because anxiety and depression overlap, there exists a common disorder. They are considered a part of a larger category called internalizing syndrome or negative affectivity, and are both variants of a single mood disorder [7]. Emotional disorders in children can cause impairment in their health, social life and academic functioning $[8,9]$. Children often use somatic complaints as a way to verbalise or express distress [10] and children with emotional disorders such as depression and anxiety more frequently report somatic complaints than children without, and somatic complaints are therefore regarded as a psychiatric symptom $[1,8]$, Headache, abdominal pain, fatigue, back pain and musculoskeletal pains are the most commonly reported somatic complaints in childhood [11]. In some epidemiological studies ADHD is significantly associated with somatic complaints [12-14].
But it is unclear how this relationship is associated, and to what extent ADHD alone is related to somatic complaints. In a study by Roessner et al. [15], they found no effects of ADHD diagnosis on the subscale of somatic complaints, but when comorbid with chronic tic disorder, an interaction effect was seen.There is a strong correlation between mental health and indicators of inequalities in wealth and income [16]. In a longitudinal study based on parental interviews by Wadsworth and Achenbach [3], on the link between SEP and psychopathology in children, they found a strong support for an association between SEP and somatic complaints, depression, anxiety, thought problems, delinquent and aggressive behaviour Heiervang and colleagues [17] described in a longitudinal study of children's mental health a significant association between low household income, low paternal education and an increased prevalence of DSM-IV disorders in children aged 7-9. The majority of research in the Nordic countries on socio-economic status and health is focused on the adult population. In these countries, where the standard of living is high, a large degree of the adult population, especially in lower SEP, struggles with mental health problems [18-20] causing functional impairments and low levels of quality of life [2122]. Mental health problems are here the leading cause of absence due to sickness and incapacity benefits [23]. Knowing the severe long-term outcomes of affected health, more research is needed on the relation between SEP and child health [24].

*Corresponding author: Maria Jensberg Leirbakk, Department of Landscape Planning, Norwegian University of Life Sciences, NMBU Ås, Akershus 1432,Norway, Tel: +4797043253; E-mail: m.j.leirbakk@medisin.uio.no

Received December 12, 2014; Accepted January 08, 2015; Published January 15,2015

Citation: Leirbakk MJ, Clench-Aas J, Ruth K. Raanaas (2014) ADHD with CoOccurring Depression/Anxiety in Children: The Relationship with Somatic Complaints and Parental Socio-Economic Position. J Psychol Abnorm Child 4: 137. doi:10.4172/2329-9525.1000137

Copyright: (c) 2015 Leirbakk MJ, et al. This is an open-access article distributed under the terms of the Creative Commons Attribution License, which permits unrestricted use, distribution, and reproduction in any medium, provided the original author and source are credited. 
The first aim of this study is to compare the risk for having somatic complaints among children with ADHD alone, depression/anxiety alone, or a co-occurrence of ADHD and depression/anxiety. We hypothesize that children with depression/anxiety alone would report higher levels of somatic complaints than children with ADHD alone. Further we hypothesize that children with ADHD and depression/ anxiety would report higher levels of somatic complaints than children with ADHD alone. The second aim of this study is to compare the risk of having ADHD and/or depression/anxiety when low parental SEP, compared to ADHD and/or depression/anxiety when average/high parental SEP. We hypothesize an increase in prevalence of ADHD and/ or depression/anxiety when decreasing SEP.

Children often use somatic complaints as a way to verbalise or express distress [25] and children with emotional disorders such as depression and anxiety more frequently report somatic complaints than children without, and somatic complaints are therefore regarded as a psychiatric symptom $[1,8]$. Headache, abdominal pain, fatigue, back pain and musculoskeletal pains are the most commonly reported somatic complaints in childhood [11]. In some epidemiological studies ADHD is significantly associated with somatic complaints [12-14]. But it is unclear how this relationship is associated, and to what extent ADHD alone is related to somatic complaints. In a study by Roessner et al. [15], they found no effects of ADHD diagnosis on the subscale of somatic complaints, but when comorbid with chronic tic disorder, an interaction effect was seen.

There is a strong correlation between mental health and indicators of inequalities in wealth and income [16].In a longitudinal study based on parental interviews by [3] on the link between SEP and psychopathology in children, they found a strong support for an association between SEP and somatic complaints, depression, anxiety, thought problems, delinquent and aggressive behaviour [17] described in a longitudinal study of children's mental health a significant association between low household income, low paternal education and an increased prevalence of DSM-IV disorders in children aged 7-9. The majority of research in the Nordic countries on socio-economic status and health is focused on the adult population. In these countries, where the standard of living is high, a large degree of the adult population, especially in lower SEP, struggles with mental health problems [18-20], causing functional impairments and low levels of quality of life $[21,22]$.

\section{Methods}

\section{Design and participants}

This study is based on data from a cross-sectional health survey of children from third grade primary school to the final year of high school in Akershus County, Oslo 2002. The study was led by the Norwegian Health Service Research Centre after initiative from municipalities in Akershus County requesting a report of health status and needs for health care services among children and youth. Akershus County surrounds Oslo and consists of 22 municipalities, covering urban, suburban and rural areas, both inland and coastline, with clear socio-economic differences. The present study is based on data from children from 8 to 13 years (3-7 grade primary school), where parents responded to the survey concerning their children. To obtain a representative sample for the County, classes were randomly selected at each school level. Parents received an information letter beforehand on the intention of the study and the need for a written consent. A total number 14,698 (78\%) parents participated. The parents received their questionnaires from the children, given them at school. It was returned to school in sealed envelopes, where it was delivered to a contact person for the municipality and from there picked up by the research administrator. Reminders were sent out once, regarding the consent form and answers to parent questionnaire. Lack of response was mainly caused by the children not being at school the day that the questionnaires were given out, which was caused by school trips, sick leaves among others. The study was anonymous and voluntary. Akershus health profile was conducted after approval from the Regional Committee for Medical and Health Research Ethics

The variables of interest in this study were ADHD, depression/ anxiety and a combination of these, used as dependent variables, and somatic complaints and SEP used as independent variables. When categorizing the two disorder groups for comparison, four groups were created. The children were categorized as having ADHD or depression/ anxiety alone, children positive to both disorders constituted a comorbid group. Children negative to both ADHD and depression/ anxiety constituted the control group.

\section{Measures}

To measure possible ADHD and depression/anxiety in the children, answers to the Strengths and Difficulties Questionnaire (SDQ) was used, which is based on the research diagnostic criteria of DSM-IV [1] SDQ is a brief screening measure that includes parent and teacher version for children 4-16 years, self-report version for adolescents 11-16 years, and a parent and preschool professional version for 3-4 year-olds. In this study, the parent version was used. SDQ contains five subscales; peer problems, hyperactivity problems, conduct problems, emotional problems and pro-social behaviour, each consisting of five questions [26].

To measure ADHD the hyperactivity-inattention subscale of the SDQ is used. Responses were summed up to a total score ranging from 0 to 10 , and recoded into three categories $(0-4=$ Low risk, $5=$ Borderline, 6-10=High risk). To measure possible ADHD, a score of "high-risk" on the SDQ hyperactivity scale (Cronbach's Alpha=0.76) score is required.

To assess depression/anxiety, scores from the emotional scale of SDQ questionnaire is used. A score among the $10 \%$ high-risk-group is required. Responses were given on a 10 point scale $(0-2=$ Low risk, $3=$ Borderline, $4-10=$ High risk) (Cronbach's Alpha $=0.66$ ). Well aware that the emotional scale includes complaints of headache, stomachache and sickness, this question was included because of the necessity to assess a total emotional scale score, and the DSM-IV definition of the disorder. Pearson correlation between "Often complains of headaches, stomach-aches or sickness" and the variable "somatic complaints" (explained further down), was 0.4.

To measure the impact of problems in everyday life, the extended version of SDQ includes an impact supplement, which was included in the survey. The DSM-IV definition of both ADHD and depression/ anxiety requires that the high-risk group answered positively to the impact question (Cronbach's Alpha on impact scale $=0.82$ ). In addition, duration is essential to characterize ADHD and depression/anxiety, classified as persistency for at least 6 months. Finally, the definition also required an impact on daily life. Impairment is needed to be present in two or more settings, with a minimum of three point score. The remaining children, $\mathrm{n}=12,127$ (94\%), negative to ADHD or depression/ anxiety constituted the control group. A total of $12,900(87,8 \%)$ parents completed the SDQ, necessary to determine possible ADHD and depression/anxiety. Cut-offs designed for the Nordic population was used on the subscales, dividing the population into three groups: $10 \%$ high-risk group corresponding to scores $>90$ th percentile, $10 \%$ borderline group and $80 \%$ low-risk group corresponding to scores $<80$ th percentile. Goodman originally designed SDQ with higher cut- 
Citation: Leirbakk MJ, Clench-Aas J, Ruth K. Raanaas (2014) ADHD with Co-Occurring Depression/Anxiety in Children: The Relationship with Somatic Complaints and Parental Socio-Economic Position. J Psychol Abnorm Child 4: 137. doi:10.4172/2329-9525.1000137

offs, but well-replicated findings of lower mean scores from the Nordic countries, required custom scores [27,28]. Mental health research in children has widely adopted SDQ as an instrument to use with satisfactory external validity against clinical diagnoses [29].

\section{Somatic complaints}

Somatic complaints included headache, abdominal pain and neck pain, and was assessed by asking the parents the frequency of their child's complaints within last six months. To be categorized as having somatic complaints, the frequency of at least one complaint, had to be "almost every week" or "almost every day". Headache, abdominal pain and neck pain were also presented individually, using the same dichotomization as for somatic complaints, where "almost every week" and "almost every day" is interpreted as having the specific complaint.

\section{Socio-economic}

Position was assessed by the parents' household income and highest completed maternal and paternal education, where education had four categories: primary, junior high school, high school and university.

\begin{tabular}{|c|c|c|}
\hline Characteristics & & $n=12,900 \%$ \\
\hline & Boy & $6,490(50.3)$ \\
\hline & Girl & $6,410(49.7)$ \\
\hline \multicolumn{3}{|c|}{ Primary school grade } \\
\hline & $3 r d$ & $2,644(20.5)$ \\
\hline & 4th & $2,658(20.6)$ \\
\hline & 5th & $2,593(20.1)$ \\
\hline & 6th & $2,516(19.5)$ \\
\hline & 7th & $2,489(19.3)$ \\
\hline \multicolumn{3}{|l|}{ Disorders } \\
\hline & Control-non of mentioned disorders & $12,127(94)$ \\
\hline & ADHD & $166(1.3)$ \\
\hline & Depression/Anxiety & $378 \quad(2.9)$ \\
\hline & ADHD and Depression/Anxiety & $229(1.8)$ \\
\hline \multicolumn{3}{|c|}{ Somatic complaints } \\
\hline & No & $11,484(89)$ \\
\hline & Yes & $743(5.8)$ \\
\hline \multicolumn{3}{|l|}{$\begin{array}{l}\text { Socio-economic } \\
\text { position }\end{array}$} \\
\hline & Low socio-economic position & $2,537(19.7)$ \\
\hline & Average/high socio-economic position & $9,675(75)$ \\
\hline
\end{tabular}

Table 1: Prevalence of 12,900 children with type of disorder, gender, primary school grade, somatic complaints and socio-economic position.
To create one variable for the socio-economic position (SEP), income was dichotomized into under the average Norwegian salary in 2002, and over the average salary. Education level was dichotomized by at least one parent with education at university level, and no education at university level for either parent. The criteria for low SEP were: income less than, or equal to, one average salary for the whole family in 2002, Norway [30] and education-level less than university for at least one parent. High/average SEP would then include income above one average salary and at least one parent with education at university level.

\section{Statistical analyses}

Analyses were all performed by The Statistical Package for the Social Sciences (SPSS) v17.0 and cases with missing responses in any of the chosen variables were removed from analysis. Associations between ADHD, depression/anxiety and other characteristics of the children and parents, were explored using bivariate and multivariate logistic regression with calculation of odds ratio (OR) and confidence intervals (95\% CI). Variables had a significant effect level at 0.05 , and were adjusted for age and school grade. Cronbach's Alpha was used on SDQ hyperactivityinattention subscale, emotional subscale and impact scale to explore internal reliability. Correlations of independent variables (gender, grade, somatic complaints and SEP) were explored using Spearman's rho.

\section{Results}

\section{Prevalence of children with ADHD, depression/anxiety and comorbidity}

Table 1 presents the prevalence of 12,900 children grade 3-7, with and without ADHD and depression/anxiety or a combination of the two. A total of 773 (6\%) children were positive to ADHD or depression/ anxiety, within this group of 773, 229 (29.6\%) children had concurrent comorbidity. According to parent-report, this study defined $1.3 \%$ (166) of the children as having ADHD alone, and 378 (2.9\%) children having depression/anxiety alone, and 229 (1.8\%) having both disorders (Table 1). Within the group of children with ADHD (395), 58\% had comorbidity with depression/anxiety. The estimate for boys exceeded that for girls, with significant gender differences for ADHD alone (OR $\left.=5.366^{* * *}\right)$, as well as for the comorbid group $\left(\mathrm{OR}=2.419^{* * *}\right)$. There was a slightly increased risk for ADHD in 4 th grade $\left(\mathrm{OR}=1.720^{*}\right)$.

\section{Somatic complaints and socio-economic position}

Table 2 presents the association between the occurrence of ADHD,

\begin{tabular}{|c|c|c|c|c|}
\hline & & $\begin{array}{c}\text { ADHD } \\
\text { OR }(95 \% \mathrm{Cl})\end{array}$ & $\begin{array}{c}\text { Depression/anxiety } \\
\text { OR }(95 \% \mathrm{Cl})\end{array}$ & $\begin{array}{l}\text { Comorbidity } \\
\text { OR }(95 \% \mathrm{Cl})\end{array}$ \\
\hline Somatic complaints & $\begin{array}{l}\text { No headache } \\
\text { Headache } \\
\text { No abdominal pain } \\
\text { Abdominal pain } \\
\text { No neck pain } \\
\text { Neck pain }\end{array}$ & $\begin{array}{l}1 . \\
1.219(0.426-3.493) \\
1 . \\
1.641(0.576-4.675) \\
1 . \\
0.626(0.085-4.625)\end{array}$ & $\begin{array}{l}1 . \\
3.771(2.611- \\
5.445) \\
\star * * \\
1 . \\
5.790(4.042- \\
8.295) \\
\star * * \\
1 . \\
2.555(1.530- \\
4.267) \\
\star * *\end{array}$ & $\begin{array}{l}1 . \\
3.335(2.039-5.455) \\
* * * \\
1 . \\
4.919(3.041-7.957) \\
* * * \\
1 . \\
4.867(2.791- \\
8489) \\
* * *\end{array}$ \\
\hline Socio-economic position & $\begin{array}{l}\text { Average/high } \\
\text { Low }\end{array}$ & $\begin{array}{l}1 . \\
1.941(1.380-2.731)\end{array}$ & $\begin{array}{l}1 . \\
2.465(1.971- \\
3.082) \\
* * *\end{array}$ & $\begin{array}{l}1 . \\
2.737(2.063-3.630) \\
\star \star \star \star\end{array}$ \\
\hline
\end{tabular}

OR=Odds-ratio, $\mathrm{Cl}=$ confidence interval. Adjusted for gender and primary school grade. Reference category is: Control - negative to ADHD or depression/anxiety. ${ }^{*} p<.05$, ${ }^{* *} p<.01,{ }^{* * *} p<.001$

Table 2: Logistic regression analysis examining the association between ADHD alone, depression/anxiety alone or comorbidity of the two, and the association of somatic complaints and socio-economic position. 


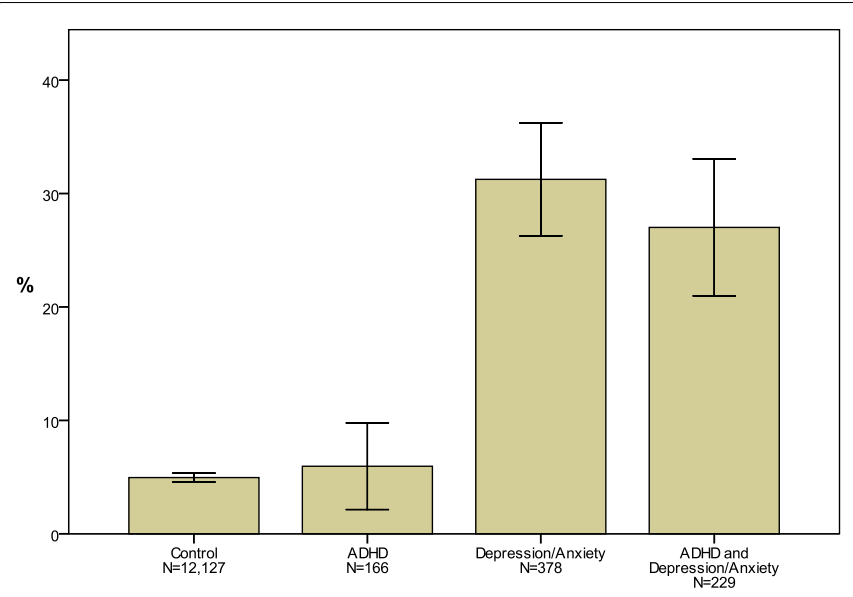

Figure 1: Prevalence (\%) of somatic complaints in children with ADHD alone, depression/anxiety alone, comorbidity of the two, and control (neither of the disorders). Error bars shows CI 95\%.

depression/anxiety and comorbidity, and somatic complaints and SEP. Figure 1 presents the prevalence of somatic complaints in children with ADHD, depression/anxiety, comorbidity and the control group having none of the diagnoses. ADHD alone was not significantly associated with any of the somatic complaints (Table 2, Figure 1). There was a strong association between depression/anxiety and all somatic complaints with abdominal pain having the highest risk: odds ratio $=5.790$. Children with depression/anxiety also had the strongest risk for having headache $(\mathrm{OR}=3.771)$. When children had both ADHD and depression/anxiety, they were strongly associated with all somatic complaints, but especially for neck pain (OR 4.867) and abdominal pain $(\mathrm{OR}=4.919)$. It was tested whether the association between depression/ anxiety and somatic complaints would be significant when the SDQ question on somatic complaints was removed from the definition of depression/anxiety. When removed, the association was still strong on all somatic complaints, and the question was kept in the definition since it is an important symptom. There was a significant association between ADHD, depression/anxiety, comorbidity and low SEP (Table 2 ), with the comorbid group being most strongly related ( OR = 2.737). Among the children with comorbidity $40.9 \%$ of the children constituted the group of low SEP, as opposed to the control group where $19.7 \%$ of children were from low SEP. The moderating effect of SEP on somatic complaints measured by comparing the odds ratio before and after controlling for SEP revealed no significant effects. However, bivariate logistic regression confirmed an association between somatic complaints and low SEP with OR being $1.713^{* * *}$, implying generally more somatic complaints in all children with parents in low SEP.

\section{Discussion}

This study suggests that somatic complaints are not more common among children having ADHD alone, but rather associated with depression/anxiety. And in addition that children from lower SEP families are more at risk for ADHD, depression or anxiety and that somatic complaint are associated with the conditions.

\section{Prevalence and identification of ADHD and depression/ anxiety}

In this study, $3.1 \%$ of the children were identified as "high-risk" for ADHD, in accordance with global and Norwegian prevalence of ADHD (American Psychiatric Association 1994) [1]. Other findings report the worldwide prevalence of ADHD to range from $2.2 \%$ to $17.8 \%$ [31] and concluded that population characteristics, methodology features, diagnostic criteria among others affect the prevalence. We have tried to be true to the diagnostic criteria of DSM-V, and considered the cultural differences by using cut-offs designed for the Norwegian population.

Findings of low prevalence (1.7\%) of ADHD in Norwegian children [17] might be caused by use of SDQ cut-offs designed for an English population. Comorbidity with ADHD and depression/anxiety of $58 \%$, is in agreement with the results of other studies [5,32] indicating the importance of comorbidity between ADHD and emotional disorders. A prevalence of $4.7 \%$ children with depression/anxiety is acceptable compared to other findings $[6,32]$.

\section{Somatic complaints in children with ADHD and/or depression/anxiety}

It was desired to clarify if the presence of significant somatic complaints in children with ADHD, actually was a result of comorbidity with depression/anxiety. Children with ADHD alone had no association with headache, neck pain or abdominal pain. If they were comorbid with depression/anxiety, they had significant associations on all somatic complaints, and even higher risk for neck pain than depression/anxiety alone. Our results stress the importance of addressing the issue of comorbidity with depression/anxiety in children with ADHD, when interpreting somatic complaints. However, we cannot clarify any causal relationship because of the study design. It is important to note the term somatic complaints, not to be confused with a somatisation syndrome, which is psychological distress or difficulty expressed through somatic symptoms without any pathological findings [10,33]. Taken into account, that some of these children actually had physiologically explained symptoms, the term somatic complaints were used.

Often, there is no medical cause for somatic complaints [34], leaving these findings in $80 \%$ of the cases to be related to anxiety, and in approximately $40 \%$ of the cases related to depression [12,14,35,36]. Both Egger and colleagues [12] and Holmberg [14] demonstrated a significant association with ADHD and abdominal pain, neither one considered the possibility of ADHD as comorbid with depression or anxiety.

\section{The inverse association when low socio-economic position}

The relationship between low parental SEP and child mental health is multifaceted [37,38]. Income is an integral, but partial determinant of health outcomes, and low parental education is not synonymous with a lack of knowledge or understanding. But this relationship is important to clarify for a well-structured public health policy to be able to prevent or intervene at an early stage. This study left no doubt concerning a higher prevalence of children having ADHD, and/or depression/ anxiety among children in families of low SEP [39]. The risk for ADHD in children with low parental SEP was almost doubled, and nearly 1.5 times more likely to meet the diagnostic criteria for depression/ anxiety compared to children with parents in an average/high SEP. The risk for having both ADHD and depression/anxiety were strongest when the child were in a family of low SEP $\left(\mathrm{OR}=2.737^{* * *}\right)$. This group also demonstrated a severe association with all somatic complaints, suggesting these children to be especially susceptible. Additionally they are as risk of limited access to health services.

There are no unambiguous etiological explanations to ADHD, the causes are multifactorial with strong genetic liability. Genetic vulnerability can be aggravated by social hardship, causing children who are born into a family with low SEP to start their life more disadvantaged compared to children born into a family of high SEP. Additionally the prevalence of ADHD is higher in the adult population with low SEP, compared to the prevalence in the population with average/high SEP. This increases the chance that a child living in a family with low SEP, inherits the disorder. 
Citation: Leirbakk MJ, Clench-Aas J, Ruth K. Raanaas (2014) ADHD with Co-Occurring Depression/Anxiety in Children: The Relationship with Somatic Complaints and Parental Socio-Economic Position. J Psychol Abnorm Child 4: 137. doi:10.4172/2329-9525.1000137

Page 5 of 6

\section{Strengths and limitations}

This study is based on information from a large representative population in Akershus County, with a response rate of $78 \%$. Selection of the ADHD and depression/anxiety group was based only on information from parents. Due to the study design, we cannot say whether or not the responding parent actually had a well medicated child diagnosed with ADHD, and therefore not among the high-risk group.

Lack of cross-informant comparisons was a limitation. Therefore, parent-report alone must be interpreted cautiously [40]. There is no knowledge on which of the parents responded. SDQ is reported to have moderate-to-high predictive sensitivity for detecting ADHD and depression/anxiety, with a good test-retest reliability [41,42]. It is important to note that defined cases of disorders were lacking clinical validity, requiring most results to be interpreted with caution. However, significant correlations reported in this study, do identify high risk groups, and by using only clinically diagnosed children, the socioeconomic factor might have been lost [3]. Families of low SEP pay fewer visits and have less access to doctors. Additionally, the help they receive is of poorer quality than higher socio-economic families [43]. This poor quality or lack of seeking help, can potentially lead to later diagnosed children and more severe outcome caused by deferred diagnosis.

\section{Implications for practice}

Health care services and professionals who work with children need to be aware that somatic complaints may be indicative of additional complications, such as depression/anxiety. Children known to have ADHD but whom also complain of somatic problems should be considered as possibly having comorbid depression/anxiety. This study also provides evidence that children from families with a low SEP are at an increased risk of suffering from these diagnoses. Both in the short and the long run children and families in need of extra support will benefit from an early detection. Due to higher risk, these children and families should be of special concern to health care services. The health care services need to offer these children and families higher priority, and better health services compared to other families [44]. Through early identification of a high-risk group, early preventive measures can be initiated [45]. Thus health care services should include routines that increase the focus on children with a high degree of somatic complaints, especially in families with low SEP.

\section{Conclusions}

Our results of prevalence of ADHD, depression/anxiety and comorbidity in children are generally consistent with those provided in previous research. However, there is a need for understanding the development of psychopathology in children, in order to provide the best treatment. It is important to be aware that ADHD is rarely homotypic when diagnosing children. This study implies that somatic complaints in children with ADHD, may be a symptom of concurrent comorbidity with depression or anxiety.

Socio-economic differences do appear when prevalence of ADHD, depression/anxiety and comorbidity in children is measured. This corresponds to findings in the adult population.

There is still a lot of research to be done to explain the symptomatic outcome of comorbid disorders in children with ADHD. The recognition of an inverse relationship in parental SEP and prevalence of both ADHD and depression/anxiety in children, may improve strategies to detect cases at an early stage and thus possibly intervene.

\section{References}

1. American Psychiatric Association, Ed. (1994). Diagnostic and Statistical Manual of Mental Disorders DSM-IV Fourt Edition. Washington, D.C.

2. Biederman, J (1995) "High risk for attention deficit hyperactivity disorder among children of parents with childhood onset of the disorder: a pilot study." The American journal of psychiatry 152: 431-435.

3. Wadsworth, M E, Achenbach T M (2005) "Explaining the link between low socioeconomic status and psychopathology: testing two mechanisms of the social causation hypothesis." Journal of consulting and clinical psychology 73 1146-1153.

4. Danckaerts M, Sonuga-Barke EJ, Banaschewski T, et al. (2010) The quality of life of children with attention deficit/hyperactivity disorder: a systematic review. European child \& adolescent psychiatry 19: 83-105.

5. 5 Costello E J (2003) "Prevalence and development of psychiatric disorders in childhood and adolescence." Archives of general psychiatry 60: 837-844.

6. Bittner A. (2007) "What do childhood anxiety disorders predict?" Journal of child psychology and psychiatry, and allied disciplines 48: 1174-1183.

7. Brady, E U , Kendall PC (1992) "Comorbidity of anxiety and depression in children and adolescents." Psychological bulletin 111: 244-255.

8. Beidel, D C (1999) "Psychopathology of childhood social phobia." Journal of the American Academy of Child and Adolescent Psychiatry. 38: 643-650.

9. Honjo S (2001) "School refusal and depression with school inattendance in children and adolescents: comparative assessment between the Children's Depression Inventory and somatic complaints." Psychiatry and clinical neurosciences. 55: 629-634.

10. Garralda M E (1996) “Somatisation in children.” Journal of child psychology and psychiatry, and allied disciplines 37: 13-33.

11. Campo, JV, Fritsch SL (1994) "Somatization in children and adolescents." Journal of the American Academy of Child and Adolescent Psychiatry 33: 12231235.

12. Egger H.L (1999)"Somatic complaints and psychopathology in children and adolescents: stomach aches, musculoskeletal pains, and headaches." Journal of the American Academy of Child and Adolescent Psychiatry 38: 852-860.

13. Graetz BW (2005) "Gender differences among children with DSM-IV ADHD in Australia." Journal of the American Academy of Child and Adolescent Psychiatry 44: 159-168.

14. Holmberg, K. and A. Hjern (2006). "Health complaints in children with attentiondeficit/hyperactivity disorder." Acta paediatrica 95(6): 664-670.

15. Roessner V (2007) "Psychopathological profile in children with chronic tic disorder and co-existing ADHD: additive effects." Journal of abnormal child psychology 35: 79-85.

16. Friedli L (2009) Mental health, resilience and inequalities, World Health Organization Office for Europe 55.

17. Heiervang, E (2007) "Psychiatric disorders in Norwegian 8- to 10-year-olds: an epidemiological survey of prevalence, risk factors, and service use." Journal of the American Academy of Child and Adolescent Psychiatry 46: 438-447.

18. Mackenbach, J P (2006) Health inequalities: Europe in profile, produced by $\mathrm{COI}$ for the Department of Health.

19. Bjelland I (2008) "Does a higher educational level protect against anxiety and depression? The HUNT study." Social science \& medicine 66: 1334-1345.

20. Dorner, TE (2011) "The impact of socio-economic status on pain and the perception of disability due to pain." European journal of pain 15: 103-109.

21. Al-Windi A (2005) "The relations between symptoms, somatic and psychiatric conditions, life satisfaction and perceived health. A primary care based study." Health and quality of life outcomes 3: 28.

22. Gjervan B (2011) "Functional Impairment and Occupational Outcome in Adults With ADHD." Journal of attention disorders. 16:544-52.

23. Harvey S B (2009) "Mental health and employment: much work still to be done." The British journal of psychiatry .the journal of mental science 194: 201-203.

24. McLaughlin K.A (2011) "Childhood socio-economic status and the onset persistence, and severity of DSM-IV mental disorders in a US national sample." Social science \& medicine 73: 1088-1096. 
Citation: Leirbakk MJ, Clench-Aas J, Ruth K. Raanaas (2014) ADHD with Co-Occurring Depression/Anxiety in Children: The Relationship with Somatic Complaints and Parental Socio-Economic Position. J Psychol Abnorm Child 4: 137. doi:10.4172/2329-9525.1000137

25. Garralda ME. (1996) Somatisation in children. Journal of child psychology and psychiatry, and allied disciplines 37: 13-33.

26. Goodman R (19970)"The Strengths and Difficulties Questionnaire: a research note." Journal of child psychology and psychiatry, and allied disciplines 38: 581 586.

27. Van Roy B (2006) "Self-reported strengths and difficulties in a large Norwegian population 10-19 years : age and gender specific results of the extended SDQquestionnaire." European child \& adolescent psychiatry 15: 189-198.

28. Heiervang, E (2008) "The Nordic advantage in child mental health: separating health differences from reporting style in a cross-cultural comparison of psychopathology." Journal of child psychology and psychiatry, and allied disciplines 49: 678-685

29. Van RoyB (2009) "Prevalence and characteristics of significant social anxiety in children aged 8-13 years: a Norwegian cross-sectional population study." Social psychiatry and psychiatric epidemiology 44: 407-415.

30. Hansen S, Skoglund T (2003) Lønnsutviklingen 1969-2002 (Development of salary 1969-2003). Økonomiske analyser 5/2003. Oslo, Statictics Norway: 44-

31. Skounti M (2007) "Variations in prevalence of attention deficit hyperactivity disorder worldwide." European Journal of Pediatrics 166: 117-123.

32. Canino G (2004) "The DSM-IV rates of child and adolescent disorders in Puerto Rico: prevalence, correlates, service use, and the effects of impairment." Archives of general psychiatry $61: 85-93$

33. Garber J (1990) "Recurrent abdominal pain in children: psychiatric diagnoses and parental psychopathology." Journal of the American Academy of Child and Adolescent Psychiatry 29: 648-656.

34. Roth-Isigkeit A (2004) "Reports of pain among German children and adolescents: an epidemiological study." Acta paediatrica 93: 258-263.
35. Campo JV (2004) "Recurrent abdominal pain, anxiety, and depression in primary care." Pediatrics 113: 817-824.

36. Jellesma F C (2006) "Somatic complaints and health care use in children: Mood, emotion awareness and sense of coherence." Social science \& medicine 63: $2640-2648$

37. Black S E (2003) Why the apple doesn't fall far: Understanding intergenerational transmission of human capital, National Bureau of Economic Research.

38. AstburyB, Leeuw FL (2010) "Unpacking black boxes: mechanisms and theory building in evaluation." American journal of evaluation 31: 363-381.

39. Fletcher JM (2014) "The effects of childhood ADHD on adult labor market outcomes." Health Econ 23: 159-181.

40. Goodman R (2003) "Using the Strengths and Difficulties Questionnaire (SDQ) to screen for child psychiatric disorders in a community sample." International review of psychiatry 15: 166-172.

41. Goodman R (1999) "The extended version of the Strengths and Difficulties Questionnaire as a guide to child psychiatric caseness and consequent burden." Journal of child psychology and psychiatry, and allied disciplines 40 : 791-799.

2. Ullebo A K (2011) "Screening for the attention deficit hyperactivity disorde phenotype using the strength and difficulties questionnaire." European child \& adolescent psychiatry 20: 451-458.

43. Sacker A (2000) "Comparing health inequality in men and women: prospective study of mortality 1986-96.” BMJ 320: 1303-1307.

44. Wallby T (2012) "Child health care utilisation in families with young or single mothers in a Swedish county." J Child Health Care 17: 17-29.

45. Klein M (2011) "Welcome back? Frequent attenders to a pediatric primary care center." J Child Health Care 15: 175-186. 